\title{
Gis 1 is required for transcriptional reprogramming of carbon metabolism and the stress response during transition into stationary phase in yeast
}

Correspondence

Nianshu Zhang

nz228@mole.bio.cam.ac.uk

Received 4 December 2008

Revised 17 February 2009

Accepted 24 February 2009

\author{
Nianshu Zhang, ${ }^{1}$ Jian $\mathrm{Wu}^{2}$ and Stephen G. Oliver ${ }^{1}$ \\ ${ }^{1}$ Cambridge Systems Biology Centre and Department of Biochemistry, University of Cambridge, \\ Sanger Building, 80 Tennis Court Road, Cambridge CB2 1GA, UK \\ ${ }^{2}$ Faculty of Life Sciences, Michael Smith Building, University of Manchester, Oxford Road, \\ Manchester M13 9PT, UK
}

\begin{abstract}
Transition from growth to the stationary phase in yeast is still poorly understood. Previously, we identified a group of yeast genes that are universally upregulated upon starvation for different macronutrients. Here, we demonstrate that the Gis 1 transcription factor and the Rim15 kinase are responsible for the upregulation of many of these genes. In chemostat cultures, gis 1 or rim 15 mutant cells are outcompeted by their wild-type parents under conditions resembling the later stages of diauxie (glucose-limiting) and post-diauxie (ethanol as a carbon source). Whilst Gis1p and Rim15p have distinct functions in gene repression, the growth defects of gis 1 or rim15 deletants can be accounted for by the overlapping functions of their protein products in promoting the expression of genes involved in glutamate biosynthesis, the glyoxylate cycle, the pentose phosphate pathway and the stress response. Further, we show that the sets of GIS1- and RIM15dependent genes and the degree of their regulation change in response to the identity of the carbon source, suggesting the likely dynamics of gene regulation exerted by Rim $15 p$ and Gis $1 p$ during different phases of the transition into stationary phase. In particular, Rim $15 p$ is required for the expression of genes involved in gluconeogenesis/glycolysis and glycerol biosynthesis only when ethanol is used as the carbon source. In agreement with this, Rim15p is shown to act in parallel with Hog $1 p$ to defend cells against osmotic stress.
\end{abstract}

\section{INTRODUCTION}

Cell growth is modulated in response to the availability of nutrients and growth factors. When grown in a glucosebased batch culture, yeast cells consume glucose and proliferate rapidly by fermentation, producing ethanol. As glucose becomes limiting, cells enter the diauxic shift and reprogramme their metabolism from fermentation to respiration. During the post-diauxic phase, the fermentation product, ethanol, is used as the carbon source. Eventually, all of the carbon sources are exhausted and yeast cells cease mitotic division and arrest at the G0 phase of the cell cycle. The key feature of entering the diauxic shift is that glucose repression is released and cells express a large set of genes involved in the utilization of alternative carbon sources, gluconeogenesis, the tricarboxylic acid (TCA) cycle, respiration and peroxisomal functions (Johnston \& Carlson, 1992). In order to survive an extended period of starvation, during the transition phase,

Abbreviations: GO, gene ontology; PDS, post-diauxic shift; UES, universally expressed at starvation.

The ArrayExpress accession number for the the microarray data generated in this study is E-MAXP- 1139. cells also acquire a set of characteristics typical of the stationary phase, including accumulation of storage carbohydrates (glycogen and trehalose), increased resistance to a variety of environmental stresses, and thickening of the cell wall (Herman, 2002).

Transcriptional reprogramming during the diauxic and post-diauxic phases involves at least one-quarter of the yeast genome (DeRisi et al., 1997) and is controlled by the Snf1 kinase pathway and a myriad of transcription factors, including the general glucose repressor Mig1p, the Hap2/3/ 4/5 complex, the Rtg3-Rtg1 complex, Adr1p, Oaf1p/Pip2p and Cat8p/Sip4p (Schuller, 2003). Two other conserved signalling pathways, Ras/cAMP and TOR, have also been implicated in the regulation of growth and entry into stationary phase in response to nutrient deprivation (Inoki et al., 2005; Thevelein \& de Winde, 1999; Wilson \& Roach, 2002; Wullschleger et al., 2006). Both PKA and TOR have been shown to regulate entry into the G0 phase of the cell cycle, converging on common targets, the Rim15 kinase and its downstream effectors, Msn2/4 and Gis1 (Swinnen et al., 2006). As a member of the PAS family, Rim15p integrates signals from TOR through the Sch9 kinase (Pedruzzi et al., 2003; Urban et al., 2007; Wanke et al., 
2008), PKA (Pedruzzi et al., 2000) and the Pho80p-Pho85p (Wanke et al., 2005) signalling pathways. It functions as a key controller of many aspects of the stationary-phase programme - including cell-cycle arrest, storage-carbohydrate accumulation and activation of Gis1p- and Msn2/4pdependent transcription (Swinnen et al., 2006).

The GIS1 gene was originally isolated as a multicopy suppressor of the $\mathrm{Gal}^{-}$phenotype of an snf1/mig1/srb8 triple mutant (Balciunas \& Ronne, 1999). It has since been shown to function as both a transcription repressor (Jang et al., 1999; Oshiro et al., 2003) and a transcription activator (Pedruzzi et al., 2000). Both transcriptional repression and activation by Gislp seem to be mediated largely via the PDS (post-diauxic shift) element present in the promoters of its target genes. By comparing the transcriptome of exponentially growing cells with that from cells exhausted for glucose in batch cultures, Cameroni et al. (2004) showed that the entire set of genes that require Rim15p for induction during the diauxic shift is included within the combined set of Gis1p- and Msn2/ $4 p$-dependent genes, suggesting that the functions of Gislp and Msn2/4p are controlled by the Rim 15 kinase. However, it remains to be found what set of genes is regulated by Gis1p and/or Rim15p in the later stages of the transition, and how they change from the diauxic (growth on limited glucose) to the post-diauxic (growth on ethanol) phase. It is also interesting to determine whether the repression functions of Gislp are also controlled by Rim15p.

In a previous study, using chemostat cultures limited for different macronutrients, we identified 17 yeast genes (SSA3, AUT7, HXT5, SML1, TBF1, MOH1, UGX2, YDL218w, YGR201c, SPG1, YHR097c, YIL055c, YIL057c, YJL144w, SPG4, UIP4 and GRE1) whose transcription is activated significantly ( $>5$-fold) by starvation for any of the macronutrients (Wu et al., 2004). We named them UES genes, for universally expressed at starvation. Here, we demonstrate that the Gis 1 transcription factor and the Rim15 kinase are responsible for activation of many of the UES genes from nutrient limitation to nutrient starvation. Gislp, as well as Rim15p, is also required for optimal cell growth in glucoseor ethanol-limited chemostat cultures, which resemble the later stages of diauxie and post-diauxie, respectively. These phenotypes can be accounted for by the overlapping functions of Gislp and Rim15p in regulating expression of genes involved in glutamate biosynthesis, the glyoxylate cycle, the pentose phosphate pathway and the stress response.

The groups of GIS1- or RIM15-dependent genes under the two carbon-limited conditions, although largely overlapping, change in accordance with the identity of the carbon source. In particular, we demonstrate that Rim 15p regulates the expression of genes involved in glycolysis/ gluconeogenesis and glycerol biosynthesis upon increased respiratory demand (ethanol as a carbon source) and that Rim15p acts in parallel with Hog1p in defending cells against hyperosmolarity. These data indicate the likely dynamics of the gene regulation exerted by Gislp and
Rim15p during the transition into the stationary phase. Furthermore, a set of stress-response genes that are upregulated in gis1 deletants are either downregulated or unchanged in rim 15 deletion mutants, suggesting that the repression function of Gislp is independent of the control exerted by Rim15p.

\section{METHODS}

Strains. Yeast deletion strains were based on BY4743 (MATa/MATa

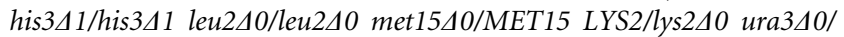
ura3 40 ) and generated by the Saccharomyces Genome Deletion Project (Winzeler et al., 1999). The hog14rim154 double mutant was generated by crossing single deletion mutants of the opposite mating types (BY4741 and BY4742) and sporulation of the resulting diploid. The genotype of the desired spore carrying both deletions is the same

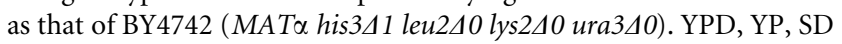
and SMM (supplemented minimal medium) media (Amberg et al., 2005) were used to grow yeast cells at 30 or $37^{\circ} \mathrm{C}$, as indicated. A stock solution $\left(1 \mathrm{mg} \mathrm{ml}^{-1}\right)$ of rapamycin (Sigma) was made up in $90 \%(\mathrm{v} / \mathrm{v})$ ethanol and $10 \%(\mathrm{v} / \mathrm{v})$ Tween 20, and used at a final concentration of $200 \mathrm{ng} \mathrm{ml}^{-1}$.

Chemostat cultures and competition experiment. Chemostat cultures were established at a dilution rate $(D)$ of $0.1 \mathrm{~h}^{-1}$ in $2 \mathrm{l}$ fermenters (Applikon Biotechnology) with both temperature $\left(30{ }^{\circ} \mathrm{C}\right)$ and $\mathrm{pH}$ (4.5) controlled. Dissolved oxygen was maintained at the maximal concentration. Glucose-, ammonium- and ethanol-limiting media were formulated, and competition experiments were performed, as reported previously (Baganz et al., 1998). Competition between the wild-type and mutant cells was carried out in chemostat cultures. Briefly, each strain was grown at steady state $\left(D=0.1 \mathrm{~h}^{-1}\right)$ in separate fermenters under carbon (glucose or ethanol) limitation. Cultures of wild-type and mutant cells were then mixed in equal volumes and growth was allowed to continue at the same dilution rate. The ratio of mutant: wild-type cells was determined from the percentage of cells displaying geneticin resistance due to their possession of the kanMX4 replacement marker.

Microarray and specific transcript analyses. Total RNA was isolated from cultures as described previously (Wu et al., 2004). Individual transcripts were assayed by Northern analysis according to Brown et al. (2001) or RT-PCR (using a kit from ABgene Inc.). Microarray experiments were carried out by using yeast genome $\mathrm{S} 98$ oligonucleotide arrays (Affymetrix, Inc.) according to the manufacturer's instructions. Normalization and statistical analyses were carried out by using Partek Genomics software (http://www.partek.com). Microarray data were generated by using two independent samples from cells grown at steady state at $D=0.1 \mathrm{~h}^{-1}$ under either glucose- or ethanol-limiting conditions. The correlation coefficients between the duplicated microarray experiments were between 0.973 and 0.996 . In compliance with MIAME guidelines, the data from this study have been deposited at the ArrayExpress repository (http://www.ebi.ac.uk/ arrayexpress/) at the European Bioinformatics Institute under accession no. E-MAXP-1139.

\section{RESULTS}

\section{Gis1p is required for induction of UES genes in response to starvation for different nutrients}

In an attempt to find possible upstream activating sequences that might help to identify the transcription 
factors involved in regulating the 17 UES genes, we used regulatory sequence analysis (RSA) tools (http://rsat.ulb.ac.be/rsat/) to retrieve over-represented oligonucleotide and dyad sequences present in their promoters. Whilst there were no significant dyad sequences found, a few hexanucleotides with high statistical significance were found. The most significant hexanucleotide, AAGGGA, is present in the promoter regions of 14 UES genes. This hexamer resembles the previously identified PDS element, which mediates transcriptional activation in response to nutritional limitation (Boorstein \& Craig, 1990). PDS element-driven expression has been shown to be strongly dependent on the Rim15 kinase and almost entirely dependent on the transcriptional activator Gis1p (Pedruzzi et al., 2000).

To confirm the dependence of the expression of these UES genes on Gis1p and Rim15p, wild-type, gis14 and rim154 cells were grown in a glucose-limited chemostat to steady state. The residual glucose concentration in the glucoselimited chemostat culture is about $30 \mathrm{mg} \mathrm{l}^{-1}$. The medium feed was then switched off and the cells were allowed to enter a glucose-starved stationary phase. As shown in Fig. 1(a), all of the transcripts analysed were strongly dependent on functional GIS1 and RIM15 genes, except for $S S A 3$, which showed a weaker and delayed transcriptional response in both gis1 14 and rim15 15 mutants. Similar results were seen when exponentially growing cells in an $\mathrm{N}$-limited chemostat were starved of ammonium (Fig. 1b). Under these conditions, abundant glucose was present for the first $12 \mathrm{~h}$ after switching off the medium feed (data not shown). The expression of these genes was also shown to be dependent on Gis1p or Rim15p in batch cultures treated with rapamycin (Fig. 1c). Induction of a similar set of genes upon rapamycin treatment has been shown previously (Pedruzzi et al., 2003). Swinnen et al. (2005) confirmed that expression of these genes is also activated when phosphate is starved, and that their activation is independent of Pho4p, but relies on the Pho81 and Rim15 kinases. Thus, Rim15p and Gis1p both play a central role in regulating the transcriptional response to different nutrient-starvation signals.

\section{GIS1 and RIM15 have overlapping functions in gene activation}

Epistasis tests have put the Rim15 kinase upstream of Gislp in the pathway that activates the nutrient-starvation response (Pedruzzi et al., 2000). To find out whether the Rim15 kinase controls all of the functions of the Gis1 transcription factor, we first determined whether all of the phenotypes revealed by a gis $1 \Delta$ strain are shared by rim154 cells. The gis1 mutant cells are temperature-sensitive when grown on rich medium (YP) with ethanol as a carbon source (Balciunas \& Ronne, 1999). Grown on supplemented minimal medium, rim15 deletants display a more severe temperature-sensitive phenotype on ethanol than do gis1 cells (Fig. 2a). On glycerol, both mutants show similar temperature-sensitive defects, compared with the wild-
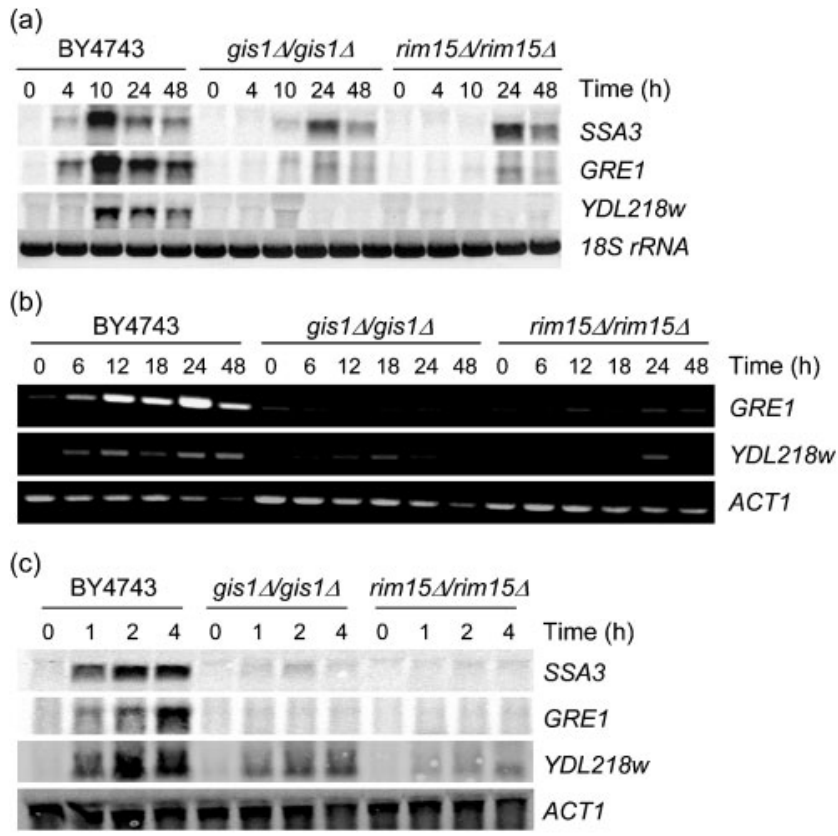

Fig. 1. Activation of UES genes is dependent on G/S1 and RIM15. (a) Glucose starvation (Northern analysis). Cells were grown to steady state in glucose-limited chemostat culture. Samples were taken 0, 4, 10, 24 and $48 \mathrm{~h}$ after the medium feed was switched off to effect glucose starvation. (b) Ammonium starvation (RT-PCR). Cells were grown to steady state in ammonium-limited chemostat culture. Samples were taken 0, 6, $12,18,24$ and $48 \mathrm{~h}$ after the medium feed was switched off to effect nitrogen starvation. (c) Rapamycin treatment (Northern analysis). Cells were grown in SD medium until mid-exponential phase $\left(\mathrm{OD}_{600}\right.$ of approx. 0.4). Samples were taken $0,1,2$ and $4 \mathrm{~h}$ after adding rapamycin.

type. When grown at $30{ }^{\circ} \mathrm{C}$, only the rim 15 mutant cells have a moderate growth defect on ethanol (Fig. 2b). No growth defects were observed for these mutants when they were grown on glucose $(2.0$ or $0.2 \%)$ at different temperatures $\left(30\right.$ or $\left.37^{\circ} \mathrm{C}\right)$.

To delineate further the phenotypic differences shown on non-fermentable carbon sources, we conducted competition experiments at a normal growth temperature $\left(30^{\circ} \mathrm{C}\right)$ under glucose- or ethanol-limiting conditions (see Methods). Both gis14 and rim154 cells were outcompeted by the wild-type under either ethanol-limited (Fig. 2c) or glucose-limited (Fig. 2d) conditions, indicating that they grew more slowly than their isogenic parents. This stringent phenotypic analysis suggests that Gislp, as well as Rim $15 p$, is functionally required for cells to adapt to both the diauxic (limited glucose as the carbon source) and the post-diauxic (ethanol as the carbon source) phases involved in the transition to stationary phase.

To find out why the gis14 and rim154 mutants were less fit than the wild-type when grown in glucose- or ethanol- 
(a)

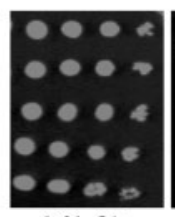

(c)
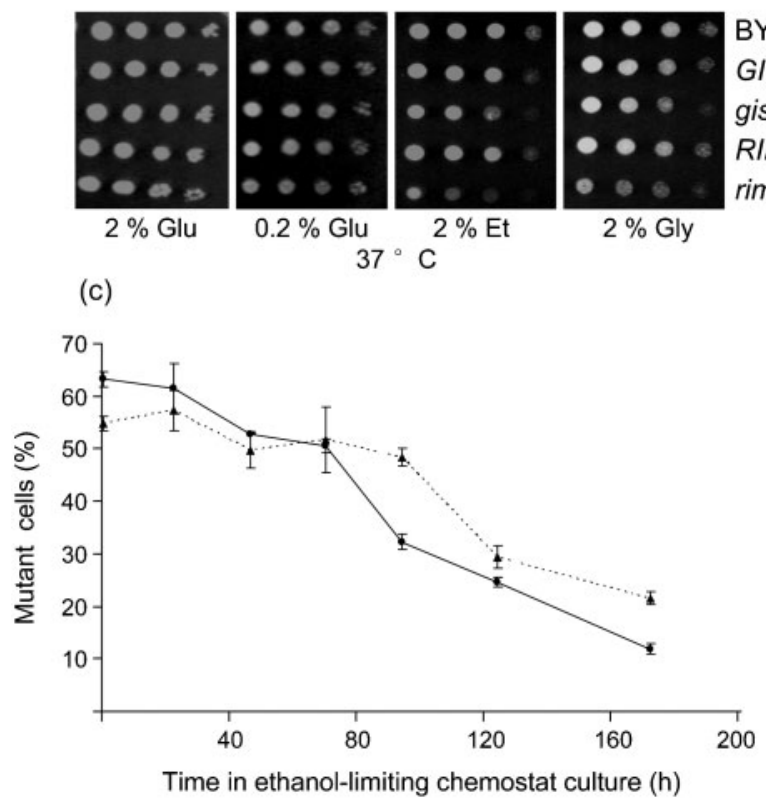

(b)

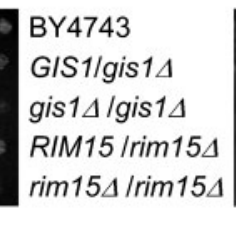

(d)
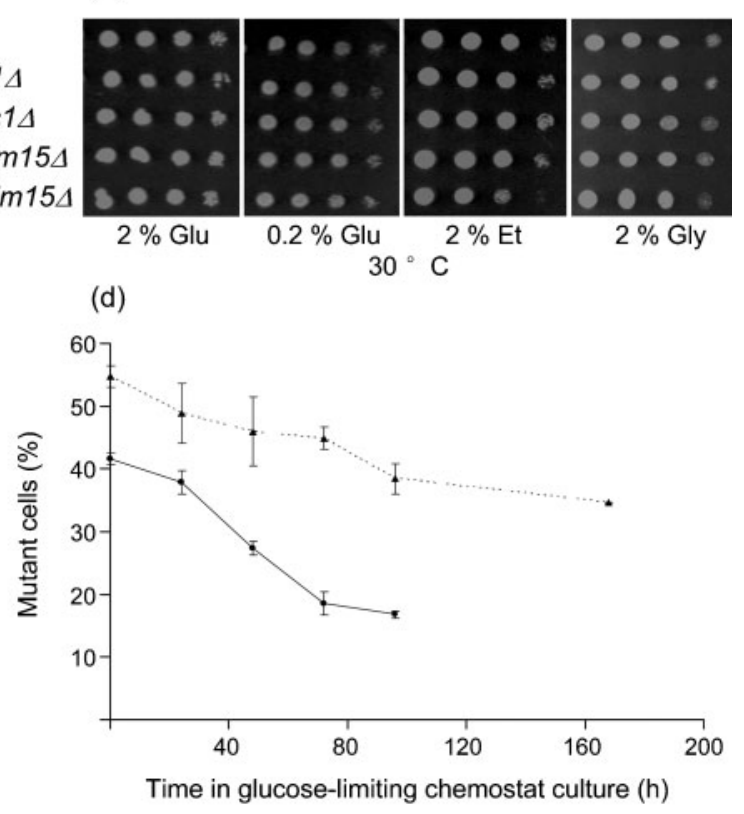

Fig. 2. Phenotypic analysis of gis $1 \Delta$ and rim154 mutants. $(a, b)$ Temperature-sensitivity test. Cells were spotted, in a 7-fold dilution series, onto SMM agar plates containing different carbon sources. Plates were incubated at either $37^{\circ} \mathrm{C}(\mathrm{a})$ or $30{ }^{\circ} \mathrm{C}$ (b) for 3-6 days. Glu, Glucose; Et, ethanol; Gly, glycerol. (c, d) Competition between the wild-type and mutant cells grown in either glucose-limited (c) or ethanol-limited (d) chemostat cultures. Samples were taken when the two strains were first mixed and at the time points indicated. Diluted cell suspensions were plated onto YPD agar and then replicated onto YPD plates containing $200 \mu \mathrm{g}$ geneticin $\mathrm{ml}^{-1}$, in order to determine the relative proportions of mutant and wild-type cells in the populations. Mean \pm SD was calculated from triplicate samples. $\boldsymbol{\Delta}$, gis 1/gis $1 ; \bullet$, rim 15/rim 15 .

limited chemostat cultures, we went on to determine whether GIS1 and RIM15 regulate the same set of genes under these conditions. Hybridization-array analyses of their transcriptomes (see Methods) demonstrated that the transcript levels of 202 ORFs were significantly different $(P<0.01)$ in the gis1/ and/or rim154 mutants compared with the wild-type grown under the same conditions. Of these transcripts, 123 changed their level by more than 1.5fold (up- or downregulated). These fell into two main functional categories: 'carbohydrate metabolism' (22 genes, $P=2.15 \times 10^{-11}$ ) and 'response to stimulus' (24 genes, $\left.P=3.64 \times 10^{-5}\right)$.

There is a significant overlap between the sets of genes whose transcription is downregulated in the two mutants compared with the wild-type, under either glucose- or ethanol-limited conditions (Fig. 3a). For example, 23 genes are downregulated more than 1.5 -fold by the gis 1 deletion when cells are growing under glucose-limited conditions. The majority of them are regulated similarly in rim154 cells (Table 1). These include genes involved in the stress response (HSP26, SIP18 and GRE1), the pentose phosphate pathway (TKL2, GND2), glutamate synthesis (IDP2, GDH3) and the glyoxylate cycle (MLS1, ICL1). Expression of the gluconeogenic genes (FBP1, PCK1) is downregulated significantly only in gis1 1 cells grown under glucose-limited conditions. These pathways are essential for cells to grow by respiration, on limited glucose or on non-fermentable carbon sources such as ethanol (Schuller, 2003). This may also explain the compromised fitness of either gis1 $\Delta$ or rim $15 \Delta$ cells in glucose- or ethanol-limited chemostat cultures (Fig. 2c, d). Of the 23 genes downregulated in the gis1 deletant, 13 have one or more nearconsensus PDS elements (WAGGGA) in their upstream regions and the pentamer 'AGGGA' is the most overrepresented motif in their promoters (Table 1). These results indicate that the Gis1 transcription factor has important roles in the activation of gene expression under conditions where there is no carbon-catabolite repression.

Among the above set of genes, expression of the majority of them (19 out of 23 ) is also induced when exponentially growing cells are treated with rapamycin (Table 1 ). The transcript level of $G D H 3$, encoding the minor form of the $\mathrm{NADP}^{+}$-dependent glutamate dehydrogenase, was also determined in gis14 and rim154 cells treated with rapamycin. As seen in Fig. 3(b), the induction of GDH3 was severely reduced in the gis1 deletant and completely abolished in the rim15 mutant. GDH3 has been shown to be repressed in cells growing by fermentation, but induced in those using respiration for growth (Avendano et al., 2005). Furthermore, a few genes sensitive to nitrogen- 
(a)

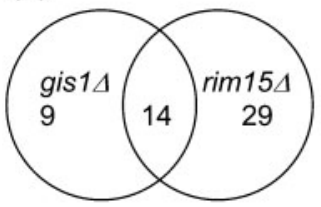

Glu-limited

(c)

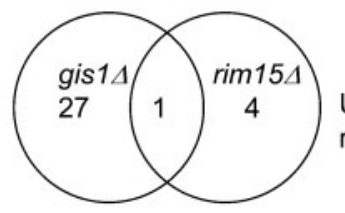

Glu-limited

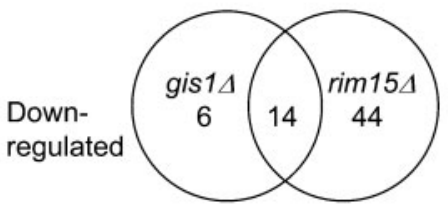

Et-limited

(b)

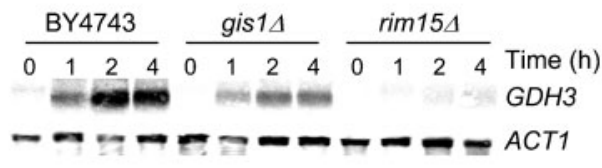

(d)

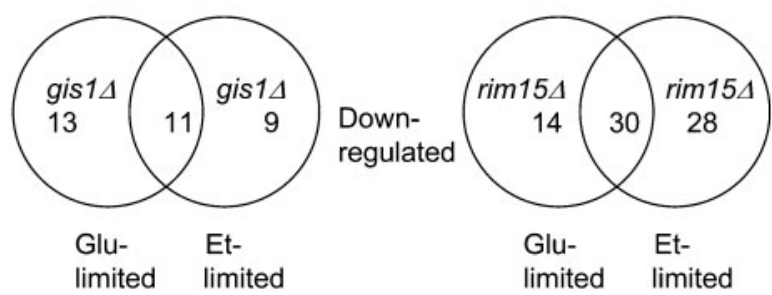

Fig. 3. Microarray analysis of gis 14 and rim 154 mutants grown in chemostat cultures. (a) Venn diagram showing the overlap between the genes downregulated in gis $1 \Delta$ and rim15 $\Delta$ cells growing in glucose (Glu)- or ethanol (Et)-limited chemostats. (b) Northern analysis of the GDH3 transcript in wild-type (BY4743), gis1 1 and rim154 cells treated with rapamycin for 0, 1, 2 and $4 \mathrm{~h}$. (c) Venn diagram showing the overlap between the genes upregulated in gis $1 \Delta$ and rim15 4 cells growing in glucose (Glu)or ethanol (Et)-limited chemostats. (d) Venn diagram showing the overlap of the downregulated genes in gis14 (left) and rim154 (right) cells.

Table 1. Genes downregulated in gis 1 deletion cells grown in glucose-limited chemostat culture

Abbreviations: BY, wild-type; gis1, gis14/gis14; rim15, rim154/rim154; G, glucose-limited; E, ethanol-limited.

\begin{tabular}{|c|c|c|c|c|c|c|c|}
\hline ORF & Description & $\begin{array}{c}\text { gis1/ } \\
\text { BY(G) }\end{array}$ & $\begin{array}{l}\operatorname{rim} 15 / \\
\mathrm{BY}(\mathrm{G})\end{array}$ & $\begin{array}{c}\text { gis1/ } \\
\text { BY(E) }\end{array}$ & $\begin{array}{l}\text { rim15/ } \\
\mathrm{BY}(\mathrm{E})\end{array}$ & $\begin{array}{c}\text { rap +/rap- } \\
\quad(2 \mathrm{~h})^{*}\end{array}$ & $\begin{array}{c}\text { PDS/ } \\
\text { STRE } \dagger\end{array}$ \\
\hline YAL062W & GDH3, $\mathrm{NADP}^{+}$-dependent glutamate dehydrogenase & -1.72 & -0.92 & -1.35 & -0.15 & 3.94 & $2 / 0$ \\
\hline YBR072W & HSP26, small heat-shock protein with chaperone activity & -0.61 & -3.03 & -0.79 & -5.25 & 2.47 & $0 / 4$ \\
\hline YBR117C & $T K L 2$, transketolase & -1.16 & -2.25 & -1.64 & -2.68 & 1.05 & $2 / 2$ \\
\hline YDL204W & RTN2, protein of unknown function & -0.61 & -0.55 & -0.53 & -0.66 & 0.58 & $1 / 1$ \\
\hline YDL223C & HBT1, substrate of the Hublp ubiquitin-like protein & -0.66 & -0.79 & -1.71 & -1.89 & 1.08 & $0 / 2$ \\
\hline YDR070C & FMP16, localized to mitochondria & -1.42 & -1.80 & -2.03 & -2.61 & 1.71 & $1 / 1$ \\
\hline YER015W & FAA2, long-chain fatty acyl-CoA synthetase & -0.61 & 0.28 & 0.09 & 0.53 & 0.63 & $0 / 0$ \\
\hline YER065C & $I C L 1$, isocitrate lyase & -1.20 & -0.71 & -0.08 & -0.02 & 0.79 & $2 / 0$ \\
\hline YFL030W & $A G X 1$, alanine : glyoxylate aminotransferase & -0.92 & -0.34 & -0.32 & 0.06 & 0.82 & $0 / 1$ \\
\hline YGL121C & GPG1, proposed $\gamma$ subunit of the heterotrimeric G protein & -1.97 & -0.83 & -1.81 & -1.36 & 2.05 & $2 / 0$ \\
\hline YGR256W & GND2, 6-phosphogluconate dehydrogenase & -2.08 & -1.81 & -0.39 & -1.01 & 1.99 & $2 / 2$ \\
\hline YIL117C & $P R M 5$, pheromone-regulated protein & -0.59 & -0.23 & 0.01 & 0.32 & 0.79 & $0 / 0$ \\
\hline YIR032C & $D A L 3$, ureidoglycolate hydrolase & -0.76 & -0.86 & -0.60 & 0.37 & 0.63 & $0 / 1$ \\
\hline YJR152W & $D A L 5$, allantoin permease & -0.67 & -0.48 & -1.71 & 1.61 & 3.92 & $0 / 0$ \\
\hline YKL163W & PIR3, O-glycosylated covalently bound cell-wall protein & -0.71 & -0.78 & 0.93 & -1.28 & 1.89 & $2 / 1$ \\
\hline YKR097W & $P C K 1$, phosphoenolpyruvate carboxykinase & -1.53 & -0.43 & -0.42 & -0.27 & -0.10 & $1 / 0$ \\
\hline YLL057C & $J L P 1, \mathrm{Fe}(\mathrm{II})$-dependent sulfonate/ $\alpha$-ketoglutarate dioxygenase & -0.80 & 0.76 & -0.50 & 0.83 & 0.35 & $0 / 1$ \\
\hline YLR174W & IDP2, cytosolic NADP-specific isocitrate dehydrogenase & -1.61 & -0.68 & -0.16 & 0.26 & 0.83 & $1 / 0$ \\
\hline YLR377C & FBP1, fructose-1,6-bisphosphatase & -1.26 & -0.26 & -0.09 & 0.53 & -0.08 & $0 / 2$ \\
\hline YML131W & Hypothetical protein & -0.61 & 0.36 & -0.47 & 0.33 & 0.09 & $0 / 1$ \\
\hline YMR175W & SIP18, induced by osmotic stress & -1.77 & -2.71 & -4.25 & -5.17 & 2.35 & $3 / 3$ \\
\hline YNL117W & MLS1, malate synthase & -1.49 & -0.86 & -0.06 & 0.05 & 3.02 & $1 / 1$ \\
\hline YPL223C & GRE1, stress-induced & -0.66 & -1.07 & -2.69 & -3.67 & 2.22 & $6 / 0$ \\
\hline
\end{tabular}

${ }^{\star}$ Comparison of transcript levels in cells treated with $(+)$ and without $(-)$ rapamycin (rap) for $2 \mathrm{~h}$. The whole dataset was published by Castrillo et al. (2007).

$\dagger$ No. of PDS elements/no. of STRE elements. 
catabolite repression, such as DAL3 and DAL5, were downregulated in the gis1 and rim15 deletants. Put together, these data suggest that RIM15 and GIS1 are important regulators of the carbon metabolism pertaining to nitrogen assimilation in the diauxic and post-diauxic phases.

\section{GIS1 and RIM15 have distinct functions in gene repression}

Gislp was shown previously to act as a transcriptional repressor of DPP1 (encoding diacylglycerol pyrophosphate phosphatase) in exponentially growing cells, and the PDS elements in the DPP1 promoter are required for this repression (Oshiro et al., 2003). As seen in Fig. 3(c), there is very little overlap between the sets of genes upregulated in the two mutants under the same carbon-limited conditions. Specifically, 28 genes are upregulated $>1.5$ fold in the gis 1 deletants compared with the wild-type when grown under glucose-limiting conditions. The transcription of only one of these genes is also upregulated in rim154 cells. In fact, the expression of the majority of them remains downregulated or unchanged in rim154 cells. A similar pattern for the same group of genes is also observed when gene expression in the two mutants is compared under ethanol limitation (Table 2). These results indicate that the function of Gislp as a transcriptional repressor is independent of that of the Rim15 kinase.

Gene ontology (GO) analysis did not reveal any significant GO term among the 28 genes upregulated in gis14 cells. Most of them encode stress-response proteins (YCR061W, GPD1, HOR2, CTT1 and DDR48) or proteins of unknown functions (BOP3, PHM8, PNS1, YCR061W, YGR066C, YHL044W, YHR140W, YJL163C, YMR173W-A and YMR181C). Interestingly, seven genes (PHM8, YGR066C, YGR248W, TPK1, MSN4, GSP2 and FRE3) are toxic to cell growth when overexpressed (Sopko et al., 2006), suggesting that one of the functions of Gis1 as a transcription repressor is to regulate properly the expression of genes important for growth. Promoter analysis was carried out to determine whether Gislp represses their expression directly. STRE elements $\left(\mathrm{AG}_{4}\right.$, targeted by Msn2/Msn4 transcription factors), rather than the PDS elements, are the most over-represented motifs in the promoters of these genes $\left(P=3.8 \times 10^{-9}\right)$. The transcription of MSN4 itself is also activated by the deletion of GIS1 and there are two near-consensus PDS elements, AAGGGA (approx. -700 to -695 and -276 to -271 ), in the promoter of MSN4. This

Table 2. List of genes upregulated in gis 1 deletion cells grown in glucose- or ethanol-limited chemostats

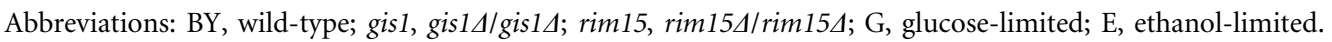

\begin{tabular}{|c|c|c|c|c|c|c|}
\hline ORF & Description & $\begin{array}{c}\text { gisl/ } \\
\text { BY(G) }\end{array}$ & $\begin{array}{l}\operatorname{rim} 15 / \\
\mathrm{BY}(\mathrm{G})\end{array}$ & $\begin{array}{c}\text { gis1/ } \\
\text { BY(E) }\end{array}$ & $\begin{array}{l}\operatorname{rim} 15 / \\
\mathrm{BY}(\mathrm{E})\end{array}$ & $\begin{array}{l}\text { STRE/ } \\
\text { PDS }^{*}\end{array}$ \\
\hline YCR061W & Protein that may be involved in response to heat and high salinity & 0.67 & -0.37 & 0.88 & -1.31 & $1 / 0$ \\
\hline YDL214C & $P R R 2$, protein kinase potentially involved in pheromone response & 1.62 & -0.25 & 0.22 & -0.16 & $2 / 2$ \\
\hline YER037W & PHM8, strong similarity to hypothetical protein YGL224c & 1.22 & -0.97 & 0.84 & -1.09 & $4 / 0$ \\
\hline YER044C & $E R G 28$, hypothetical protein; endoplasmic reticulum membrane protein & 0.60 & 0.32 & 0.31 & -0.52 & $0 / 0$ \\
\hline YGR066C & Similarity to hypothetical protein YBR105c & 0.92 & -0.17 & 0.99 & -0.39 & $1 / 1$ \\
\hline YGR088W & CTT1, cytoplasmic catalase $\mathrm{T}$ & 1.24 & -0.50 & 0.45 & -2.24 & $4 / 0$ \\
\hline YGR248W & Possible 6-phosphogluconolactonase & 0.60 & -0.48 & 0.37 & -1.01 & $1 / 2$ \\
\hline YHL044W & Member of the duplication (DUP240) family & 0.64 & -0.12 & 1.05 & -0.12 & $0 / 0$ \\
\hline YHR016C & YSC84, protein involved in the organization of the actin cytoskeleton & 0.64 & 0.19 & 0.75 & -0.18 & $1 / 1$ \\
\hline YHR140W & Protein that contains six predicted transmembrane segments & 0.63 & -0.08 & 0.19 & -0.26 & $1 / 1$ \\
\hline YMR105C & PGM2, phosphoglucomutase & 0.76 & -0.11 & 0.26 & -1.61 & $5 / 0$ \\
\hline YMR173W & DDR48, induced by heat shock, DNA damage or osmotic stress & 0.81 & -0.75 & 0.57 & -1.08 & $2 / 1$ \\
\hline YMR173W-A & Protein of unknown function & 0.73 & -0.57 & 0.29 & -1.03 & $2 / 0$ \\
\hline YMR181C & Protein of unknown function & 0.62 & 0.02 & 0.81 & 0.28 & $2 / 0$ \\
\hline YMR319C & FET4, low-affinity $\mathrm{Fe}(\mathrm{II})$-transport protein & 1.50 & 0.07 & 0.34 & -0.05 & $2 / 2$ \\
\hline YNL042W & $B O P 3$, bypass of PAM1; protein of unknown function & 0.73 & -0.17 & 0.59 & -0.13 & $2 / 0$ \\
\hline YOR161C & PNS1, mitochondrial protein of unknown function & 1.75 & -0.19 & 1.74 & -0.50 & $2 / 1$ \\
\hline YOR185C & GSP2, Ran, a GTP-binding protein member of the ras superfamily & 0.75 & -0.04 & 1.08 & -0.37 & $2 / 1$ \\
\hline YOR381W & FRE3, similar to FRE2; ferric reductase & 0.93 & 0.15 & 0.03 & -0.36 & $0 / 2$ \\
\hline
\end{tabular}

${ }^{\star}$ No. of STRE elements/no. of PDS elements. 
suggests that, at least in actively growing cells, Gis1p may target either the PDS elements directly, or the STRE elements indirectly via Msn4p, to repress the expression of a set of stress-response genes.

\section{Gene regulation exerted by Gis1p and Rim15p changes in response to the identity of the carbon source}

Deletion of either GIS1 or RIM15 leads to growth-fitness defects in both the glucose- and ethanol-limited media (Fig. 2c, d), suggesting that GIS1 and RIM15 control different stages of the transition to stationary phase. Correspondingly, $>50 \%$ of the genes downregulated in the glucose-limited mutant cells are regulated similarly when the deletants are grown in ethanol-limited chemostats (Fig. 3d). However, the degree of regulation for most of these genes changes in response to the identity of the carbon source. For example, the effect of gis 1 or rim 15 deletions on the expression of three stress-response genes (HSP26, SIP18 and GRE1) is more pronounced under ethanol-limiting conditions (Table 1). Moreover, some of the GIS1- or RIM15-dependent genes are regulated specifically under either glucose- or ethanol-limiting conditions. Thus, the expression of PIS1 (phosphatidylinositol synthase), CDA1 (chitin deacetylase) and a few others is not affected significantly by the deletion of GIS1 in glucose-limited cells, but loss of GIS1 leads to downregulation under the ethanol-limited condition. Similarly, the expression of 28 genes was downregulated in the rim154 strain only when cells were grown in ethanollimited chemostat cultures. This set of 28 includes genes involved in defence against oxidative damage (CTT1 and $A H P 1)$, glycolysis/gluconeogenesis (CDC19, ENO1, TDH3, GPM1 and PGM2) and glycerol biosynthesis (HOR2, RHR2 and GPD1). This indicates that Rim15p controls the expression of both the damage-defence genes and glycerol-biosynthetic genes in response to increased oxidative stress when cells are grown on ethanol as a carbon source. These data also suggest that Rim15 and Gis1 regulate the expression of different sets of genes at different stages of the transition to stationary phase.

Production of glycerol is required to protect yeast cells from oxidative stress (Pahlman et al., 2001), which is generally triggered by reactive oxygen species produced as a result of an increase in mitochondrial respiratory activity (Costa \& Moradas-Ferreira, 2001). Glycerol production in yeast is also known to have fundamental physiological roles in adaptive responses to osmoregulation and anaerobic redox control (Maeda et al., 1994). The involvement of Rim $15 p$ in glycerol biosynthesis suggests that Rim 15p may be required for cells to defend themselves against osmotic stress. As shown in Fig. 4, whilst the hog1 mutants were sensitive to osmotic stress at all concentrations of sorbitol tested, the rim 15 deletants displayed a growth defect only at the high concentration of sorbitol $(2.8 \mathrm{M})$. The double mutant displayed a more severe growth defect at the low concentration of sorbitol $(0.7 \mathrm{M})$ than either single mutant when incubation was extended to 5 days. These data suggest that Rim15p may be required to coordinate central carbon metabolism and glycerol production in response to different stresses and that Rim15 and Hog 1 act in parallel to defend cells against osmotic shock.

Previously, Cameroni et al. (2004) identified a number of genes that are regulated by RIM15, GIS1 and MSN2/4 during the diauxic shift by comparing the transcriptome from cells in the exponential phase of batch culture with those from cells that have just exhausted the glucose in the medium. Interestingly, there is very little overlap between the gene sets identified in their experiments and those that we have uncovered. For example, among the 57 genes shown to be downregulated in rim15 mutant cells by Cameroni et al. (2004), only four of them are regulated similarly in the same cells grown in glucose- or ethanollimited chemostats. In fact, the majority are not regulated significantly by RIM15 under either glucose- or ethanollimiting conditions (data not shown). Similarly, no significant correlation is observed between the set of GIS1-dependent genes revealed by Cameroni et al. (2004) and those shown in our study (data not shown). Whilst Cameroni et al. (2004) studied the effects of deletions of RIM15, GIS1 and MSN2/4 on gene expression during early diauxie, the physiological conditions of the glucose- and ethanol-limited chemostat cultures used in this study are more similar to the later stages of diauxie and post-diauxie, respectively. These data may further support the notion that Rim15p and its downstream effectors regulate the expression of changing sets of genes during different phases of the transition into stationary phase.

\section{DISCUSSION}

We have demonstrated, by phenotypic and transcriptomic analyses, that both Gis1p and Rim15p are required for cells

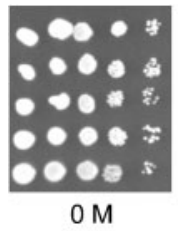

2 days
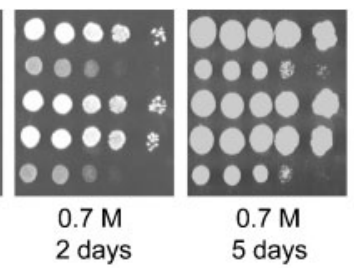

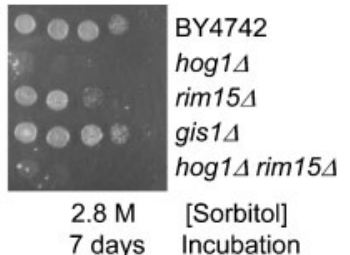

Fig. 4. Phenotypic analysis of rim15-deletion cells exposed to hyperosmolarity. Cells were spotted, in a 7-fold dilution series, onto YPD plates and incubated at $30{ }^{\circ} \mathrm{C}$. Sorbitol concentration and incubation time are indicated. 
to grow on limited glucose or ethanol as a carbon source. Such conditions resemble the later stages of diauxie and post-diauxie, respectively. Both GIS1 and RIM15 are also required for activation of a number of UES genes when cells are starved of different macronutrients. Deletion of either of them has a deleterious effect on cell survival in the stationary phase (Pedruzzi et al., 2000). Overall, the data demonstrate that Rim15p and Gis1p both participate in an important pathway that regulates yeast gene expression during the transitions that precede entry into the stationary phase.

Compared with the transcriptome analyses conducted during the diauxic shift of yeast in batch culture (Cameroni et al., 2004), our chemostat studies have shown very little correlation between the two sets of genes regulated by either RIM15 or GIS1 (data not shown). The sets of RIM15- or GIS1-dependent genes and the degree of their regulation change according to the identity of the limiting carbon source (Fig. 3d). These data suggest that there are likely dynamic changes of gene regulation exerted by Gislp and Rim15p during different phases of the shift into the stationary phase. Whilst Gislp and Rim15p have overlapping functions in regulating the expression of genes involved in central carbon metabolism and the stress response, we have revealed that a set of genes repressed by Gislp was either downregulated or unchanged in rim154 cells, suggesting that the action of Gislp as a transcription repressor is independent of the control of the Rim15 kinase. This repression activity of Gislp may be mediated directly, by its binding to PDS elements, or indirectly via Msn4p given that STRE elements are the most-represented motifs in the promoters of these genes and the expression of MSN4 is, itself, regulated by GIS1 (Table 2).

However, it cannot be ruled out that GIS1 may mediate gene activation or repression by binding to STRE elements directly. Firstly, among the downregulated genes in the gis1 mutants, not all contain PDS elements and the majority also have STRE elements in their promoters (Table 1). Secondly, $95 \%$ of Gislp-dependent genes are included within the larger set of Msn2/4p-dependent genes (Cameroni et al., 2004). Furthermore, Gislp and Msn2/ $4 \mathrm{p}$ were demonstrated to have partially redundant functions in regulating the expression of genes containing only PDS or STRE elements in their promoters (Roosen et al., 2005). It will be interesting to find out how GIS1 and MSN2/4 cooperate to both activate and repress gene transcription under the same physiological conditions.

Rim15p was shown to control the expression of several genes that are classically involved in defence against oxidative stress (Cameroni et al., 2004). In this study, we have extended the understanding of the role of Rim 15p in the oxidative-stress response by demonstrating that it regulates the expression of glycerol-biosynthetic genes under conditions of increased mitochondrial respiratory activity (Fig. 3d). These data suggest that Rim15p coordinates carbon metabolism and the oxidative-stress response during the transition from nutrient limitation to stationary phase. A number of studies have demonstrated that the Rim15 kinase receives nutritional signals from both the PKA (Pedruzzi et al., 2000) and TOR (Pedruzzi et al., 2003; Urban et al., 2007; Wanke et al., 2008) signalling pathways. The PAS domain at the $\mathrm{N}$-terminal region of Rim15p was suggested to sense the oxidative-stress signal (Cameroni et al., 2004). However, how Rim15p integrates both the nutrient- and oxidative-stress signals and how the activity of its downstream effectors is regulated to coordinate metabolism and stress responses remain to be determined. Recently, the general stress-response transcription factor Msn2/4p was shown to receive signals from both Hog1 and other pathways to activate the expression of a large number of genes upon osmotic shock (Capaldi et al., 2008). Given that deletion of both RIM15 and HOG1 renders cells more sensitive to hyperosmotic conditions than either of the single mutants (Fig. 4) and that the expression of Msn2/4p-dependent genes is also controlled by Rim15p under a number of physiological conditions (Cameroni et al., 2004; Roosen et al., 2005; Swinnen et al., 2006), it is tempting to suggest that the Hog 1 and Rim 15 kinases function cooperatively to activate the osmoticstress response programme.

\section{ACKNOWLEDGEMENTS}

We would like to thank Andy Hayes and Leanne Wardleworth for assistance with microarray experiments. This work was supported by BBSRC grants awarded to S. G. O.

\section{REFERENCES}

Amberg, D., Burke, D. J. \& Strathern, J. N. (2005). Methods in Yeast Genetics. Cold Spring Harbor, NY: Cold Spring Harbor Laboratory.

Avendano, A., Riego, L., DeLuna, A., Aranda, C., Romero, G., Ishida, C., Vazquez-Acevedo, M., Rodarte, B., Recillas-Targa, F. \& other authors (2005). Swi/SNF-GCN5-dependent chromatin remodelling determines induced expression of GDH3, one of the paralogous genes responsible for ammonium assimilation and glutamate biosynthesis in Saccharomyces cerevisiae. Mol Microbiol 57, 291-305.

Baganz, F., Hayes, A., Farquhar, R., Butler, P. R., Gardner, D. C. J. \& Oliver, S. G. (1998). Quantitative analysis of yeast gene function using competition experiments in continuous culture. Yeast 14, 14171427.

Balciunas, D. \& Ronne, H. (1999). Yeast genes GIS1-4: multicopy suppressors of the $\mathrm{Gal}^{-}$phenotype of snf1 migl srb8 cells. Mol Gen Genet 262, 589-599.

Boorstein, W. R. \& Craig, E. A. (1990). Regulation of a yeast $H S P 70$ gene by a cAMP responsive transcriptional control element. EMBO J 9, 2543-2553.

Brown, A. J., Planta, R. J., Restuhadi, F., Bailey, D. A., Butler, P. R., Cadahia, J. L., Cerdan, M. E., De Jonge, M., Gardner, D. C. J. \& other authors (2001). Transcript analysis of 1003 novel yeast genes using high-throughput northern hybridizations. EMBO J 20, 3177-3186.

Cameroni, E., Hulo, N., Roosen, J., Winderickx, J. \& De Virgilio, C. (2004). The novel yeast PAS kinase Rim 15 orchestrates G0-associated antioxidant defense mechanisms. Cell Cycle 3, 462-468. 
Capaldi, A. P., Kaplan, T., Liu, Y., Habib, N., Regev, A., Friedman, N. \& O'Shea, E. K. (2008). Structure and function of a transcriptional network activated by the MAPK Hog1. Nat Genet 40, 1300-1306.

Castrillo, J. I., Zeef, L. A., Hoyle, D. C., Zhang, N., Hayes, A., Gardner, D. C. J., Cornell, M. J., Petty, J., Hakes, L. \& other authors (2007). Growth control of the eukaryote cell: a systems biology study in yeast. J Biol 6, 4 .

Costa, V. \& Moradas-Ferreira, P. (2001). Oxidative stress and signal transduction in Saccharomyces cerevisiae: insights into ageing, apoptosis and diseases. Mol Aspects Med 22, 217-246.

DeRisi, J. L., Iyer, V. R. \& Brown, P. O. (1997). Exploring the metabolic and genetic control of gene expression on a genomic scale. Science 278, 680-686.

Herman, P. K. (2002). Stationary phase in yeast. Curr Opin Microbiol 5, 602-607.

Inoki, K., Ouyang, H., Li, Y. \& Guan, K. L. (2005). Signaling by target of rapamycin proteins in cell growth control. Microbiol Mol Biol Rev 69, 79-100.

Jang, Y. K., Wang, L. \& Sancar, G. B. (1999). RPH1 and GIS1 are damage-responsive repressors of PHR1. Mol Cell Biol 19, 7630-7638.

Johnston, M. \& Carlson, M. (1992). Regulation of carbon and phosphate utilization. In The Molecular and Cellular Biology of the Yeast Saccharomyces cerevisiae Gene Expression, pp. 193-281. Edited by E. W. Jones, J. R. Pringle \& J. R. Broach. Cold Spring Harbor, NY: Cold Spring Harbor Laboratory.

Maeda, T., Wurgler-Murphy, S. M. \& Saito, H. (1994). A twocomponent system that regulates an osmosensing MAP kinase cascade in yeast. Nature 369, 242-245.

Oshiro, J., Han, G. S., Iwanyshyn, W. M., Conover, K. \& Carman, G. M. (2003). Regulation of the yeast DPP1-encoded diacylglycerol pyrophosphate phosphatase by transcription factor Gislp. J Biol Chem 278, 31495-31503.

Pahlman, A. K., Granath, K., Ansell, R., Hohmann, S. \& Adler, L. (2001). The yeast glycerol 3-phosphatases Gpp1p and Gpp2p are required for glycerol biosynthesis and differentially involved in the cellular responses to osmotic, anaerobic, and oxidative stress. J Biol Chem 276, 3555-3563.

Pedruzzi, I., Burckert, N., Egger, P. \& De Virgilio, C. (2000). Saccharomyces cerevisiae Ras/cAMP pathway controls post-diauxic shift element-dependent transcription through the zinc finger protein Gis1. EMBO J 19, 2569-2579.

Pedruzzi, I., Dubouloz, F., Cameroni, E., Wanke, V., Roosen, J., Winderickx, J. \& De Virgilio, C. (2003). TOR and PKA signaling pathways converge on the protein kinase Rim15 to control entry into $\mathrm{G}_{0}$. Mol Cell 12, 1607-1613.

Roosen, J., Engelen, K., Marchal, K., Mathys, J., Griffioen, G., Cameroni, E., Thevelein, J. M., De Virgilio, C., De Moor, B. \&
Winderickx, J. (2005). PKA and Sch9 control a molecular switch important for the proper adaptation to nutrient availability. Mol Microbiol 55, 862-880.

Schuller, H. J. (2003). Transcriptional control of nonfermentative metabolism in the yeast Saccharomyces cerevisiae. Curr Genet 43, 139160.

Sopko, R., Huang, D., Preston, N., Chua, G., Papp, B., Kafadar, K., Snyder, M., Oliver, S. G., Cyert, M. \& other authors (2006). Mapping pathways and phenotypes by systematic gene overexpression. Mol Cell 21, 319-330.

Swinnen, E., Rosseels, J. \& Winderickx, J. (2005). The minimum domain of Pho81 is not sufficient to control the Pho85-Rim15 effector branch involved in phosphate starvation-induced stress responses. Curr Genet 48, 18-33.

Swinnen, E., Wanke, V., Roosen, J., Smets, B., Dubouloz, F., Pedruzzi, I., Cameroni, E., De Virgilio, C. \& Winderickx, J. (2006). Rim15 and the crossroads of nutrient signalling pathways in Saccharomyces cerevisiae. Cell Div 1, 3.

Thevelein, J. M. \& de Winde, J. H. (1999). Novel sensing mechanisms and targets for the cAMP-protein kinase A pathway in the yeast Saccharomyces cerevisiae. Mol Microbiol 33, 904-918.

Urban, J., Soulard, A., Huber, A., Lippman, S., Mukhopadhyay, D., Deloche, O., Wanke, V., Anrather, D., Ammerer, G. \& other authors (2007). Sch9 is a major target of TORC1 in Saccharomyces cerevisiae. Mol Cell 26, 663-674.

Wanke, V., Pedruzzi, I., Cameroni, E., Dubouloz, F. \& De Virgilio, C. (2005). Regulation of G0 entry by the Pho80-Pho85 cyclin-CDK complex. EMBO J 24, 4271-4278.

Wanke, V., Cameroni, E., Uotila, A., Piccolis, M., Urban, J., Loewith, R. \& De Virgilio, C. (2008). Caffeine extends yeast lifespan by targeting TORC1. Mol Microbiol 69, 277-285.

Wilson, W. A. \& Roach, P. J. (2002). Nutrient-regulated protein kinases in budding yeast. Cell 111, 155-158.

Winzeler, E. A., Shoemaker, D. D., Astromoff, A., Liang, H., Anderson, K., Andre, B., Bangham, R., Benito, R., Boeke, J. D. \& other authors (1999). Functional characterization of the $S$. cerevisiae genome by gene deletion and parallel analysis. Science 285, 901-906.

Wu, J., Zhang, N., Hayes, A., Panoutsopoulou, K. \& Oliver, S. G. (2004). Global analysis of nutrient control of gene expression in Saccharomyces cerevisiae during growth and starvation. Proc Natl Acad Sci U S A 101, 3148-3153.

Wullschleger, S., Loewith, R. \& Hall, M. N. (2006). TOR signaling in growth and metabolism. Cell 124, 471-484.

Edited by: M. Molina 